\title{
Atomic force microscopy study of cellulose surface interaction controlled by cellulose binding domains
}

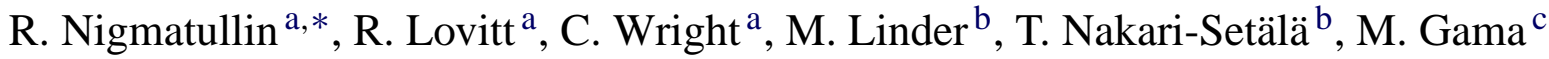 \\ ${ }^{a}$ Centre for Complex Fluids Processing, School of Engineering, University of Wales Swansea, Singleton Park, Swansea SA2 8PP, UK \\ ${ }^{\mathrm{b}}$ VTT Biotechnology and Food Research, P.O. Box 1500, FIN-02044 VTT, Finland \\ ${ }^{c}$ Centro de Engenharia Biológica-IBQF, Universidade do Minho, Largo do Paço, 4719 Braga, Portugal
}

Received 3 April 2003; accepted 9 February 2004

\begin{abstract}
Colloidal probe microscopy has been used to study the interaction between model cellulose surfaces and the role of cellulose binding domain (CBD), peptides specifically binding to cellulose, in interfacial interaction of cellulose surfaces modified with CBDs.

The interaction between pure cellulose surfaces in aqueous electrolyte solution is dominated by double layer repulsive forces with the range and magnitude of the net force dependent on electrolyte concentration. AFM imaging reveals agglomeration of CBD adsorbed on cellulose surface. Despite an increase in surface charge owing to CBD binding to cellulose surface, force profiles are less repulsive for interactions involving, at least, one modified surface. Such changes are attributed to irregularity of the topography of protein surface and non-uniform distribution of surface charges on the surface of modified cellulose.

Binding double CBD hybrid protein to cellulose surfaces causes adhesive forces at retraction, whereas separation curves obtained with cellulose modified with single CBD show small adhesion only at high ionic strength. This is possibly caused by the formation of the cross-links between cellulose surfaces in the case of double CBD.
\end{abstract}

(C) 2004 Elsevier B.V. All rights reserved.

Keywords: AFM; Colloid probe; Cellulose surface; Cellulose binding domain; Force measurements

\section{Introduction}

Cellulose is the most abundant natural polymer and is extensively used as a renewable raw material for many products in paper, wood, and textile manufacturing. A distinctive structural feature of cellulose materials is heterogeneity owing to its fibre structure. Cellulose materials form three-dimensional networks with inclusion of other molecular and particulate additives. The insolubility of cellulose means that technological processes are also based on colloid systems, for example, fibre suspension in papermaking. Therefore, interfacial interactions involving cellulose are of particular interest for cellulose material production and utilization. These interactions not only govern processes of manufacturing, recycling, coating, etc. but also determine the physical properties of cellulose-based products.

\footnotetext{
* Corresponding author.

E-mail address: r.nigmatullin@swansea.ac.uk (R. Nigmatullin).
}

Recent advances in force measurements using the surface force apparatus (SFA) [1] and the atomic force microscope (AFM) $[2,3]$ permit the experimental study of surface and inter-particle forces in controllable environments. These techniques have been used to measure interactions of cellulose surfaces [4-9] aiming mostly to increase the insight into interfacial phenomena in traditional cellulose applications such as papermaking. With growing interest in new cellulose applications often involving biomolecules or even cells and tissues (drug delivery devices [10], affinity separation [11], tissue engineering [12]) surface force measurements are becoming important to advance understanding of interfacial phenomena in such complex multi-component systems based on cellulose polymers.

Non-covalent specific binding plays an important role in biological processes and is increasingly applied in biotechnology. Specific interactions involving a cellulose matrix can be mediated by cellulose binding domains (CBDs). These are auxiliary domains that have been found in cellulose- and hemicellulose-degrading enzymes and 
provide enzyme binding to the cellulose [13]. High affinity and stability of specific CBD/cellulose binding have stimulated endeavors to exploit such interactions for cellulose surface modification and biotechnological applications of cellulose. Biotechnological applications also benefit from the fact that isolated CBDs preserve their high affinity for cellulose. In addition, CBDs can be fused with enzymes, proteins or polypeptides (using recombinant DNA technology) providing hybrid biomolecules, which are capable to selective binding to cellulose matrix [14].

CBDs and hybrid proteins containing CBD has been used for modification of cellulose fibers. For example, CBD, showing non-hydrolytic disruptive activity on cellulose fibers, causes a release of microfibrils as small particles and prevents the flocculation of microcrystalline bacterial cellulose [15-18]. These improve processability of the cellulose fibers and paper strength. Paper strength has been improved by the treatment of cellulose fibers with cellulose cross-linking protein (CCP), which consists of two CBD fused together [19]. Further, such a treatment increases hydrophobicity of paper surface. Structural modification of cellulose with isolated CBD also alters dyeing characteristics of cellulose fibers [20]. More benefits for products of paper and textile manufacturing are expected with use of hybrid proteins containing CBD [14].

CBD also provide a new class of affinity tags increasing the range of application of relatively cheap cellulose materials in bioreactors, bioseparation, diagnosis, etc. Recombinant cells with surface-expressed CBD or hybrid enzymes/proteins containing CBD are easily and reliably bound to cellulose supports, thus providing a very simple and economical way of whole-cell or protein immobilization [14,21-23]. On the other hand, use of CBD families, which reversibly bind to cellulose, in protein engineering provide specific tags for bioseparation on cellulose matrix by affinity chromatography [24].

The binding of CBD to cellulose alters the cellulose surface chemistry and thus changes the interfacial phenomena in systems exploiting the properties of cellulose. In this paper, we present an AFM study of the interfacial interactions between cellulose surfaces in a range of aqueous environment in the presence of two CBDs of the family I, which are strongly affine to crystalline and semi-crystalline cellulose with affinity constant exceeding $1 \times 10^{-5} \mathrm{M}^{-1}$ [24].

\section{Materials and methods}

\subsection{Chemicals}

Sodium chloride ( $\mathrm{NaCl}$, certified ACS grade) was used as received from Aldrich. Filtered $(0.22 \mu \mathrm{m}$ filter) MQ water ('Milli-Q'; resistivity, $18.2 \mathrm{MV} \mathrm{cm}$, Millipore) was used for all aqueous solutions and for rinsing. The water $\mathrm{pH}$, when equilibrated with the atmosphere, was about 5.6 due to dissolved $\mathrm{CO}_{2}$.

\subsection{CBD samples}

Two types of CBD used in the work are derived from Trichoderma reesei. A single cellulose binding domain coded as CBD was prepared by enzymatic hydrolysis of fungal cellulases according to the protocol described elsewhere [25]. A crude cellulase preparation (Celluclast, Novo Nordisk) was hydrolysed with papain. After digestion CBDs were obtained by ultrafiltration of the digestion mixture through a hollow fibre cartridge with a $10 \mathrm{kDa}$ nominal cut off. This procedure allowed CBD isolation with a yield of about $80 \%$.

The second type of CBD was a double CBD, coded as HFBI-CBD. It is a recombinant chimeric protein consisting of two CBDs from $T$. reesei, which preparation is described by Linder et al. [26]. To the $\mathrm{N}$ terminus of this construct the hydrophobin protein HFBI also from $T$. reesei was fused in order to facilitate the protein purification [27]. The sequence of the HFBI tag is: SNGNGNVCPP GLFSNPQCCA TQVLGLIGLD CKVPSQNVYD GTDFRNVCAK TGAQPLCCVA PVAGQALLCQ TAVGA. This sequence is followed by a short linker: PGASTSTGMGPGG, after which the CBD of cellobiohydrolase II (CBHII CBD) comes: QACSSVWGQC GGQNWSGPTC CASGSTCVYS NDYYSQCL. To this, a linker PGANPPGTTT TSRPATTTGSSPGP connects the C-terminal of the CBD of cellobiohydrolase I (CBHI CBD) which has the sequence: TQSHYGQCGG IGYSGPTVCA SGTTCQVLNP YYSQCL. The protein was produced in $T$. reesei using the $c b h 1$ promoter. The protein was purified from the culture supernatant using the HFBI tag by surfactant extraction as described in [27]. Briefly, 2\% Berol 532 (Akzo-Nobel, Sweden) was added to the supernatant. The upper phase was collected and an equal volume of water was added together with a five-fold volume of isobutanol. The aqueous phase contained the HFBI-DCBD, which was further purified by reversed-phase chromatography on a $\mathrm{C} 4$ Vydac column (Vydac, USA) using $0.1 \%$ trifluoro acetic acid as the equilibration buffer and eluting with a gradient of acetonitrile containing $0.1 \%$ trifluoro acetic acid. The HFBI-DCBD fraction was then lyophilised. The product showed as a single band in SDS-PAGE at about $20 \mathrm{kDa}$ and reacted with polyclonal antibodies against HFBI and a monoclonal antibody (CI-89) against the CBHI CBD.

\subsection{Cellulose sample preparation}

Force measurements were carried out between planar cellulose surface and a spherical cellulose probe. A supported film of regenerated cellulose was prepared by casting a cellulose acetate (Cellulose acetate 398-10, Eastman Company) solution on a porous polypropylene support followed by alkaline saponification. A filtered $16 \mathrm{wt} . \%$ solution of cellulose acetate in acetone was spread onto a support as an uniform layer of thickness $500 \mu \mathrm{m}$ using a knife with a calibrated slit. The solvent was completely evaporated at $40^{\circ} \mathrm{C}$ for $20 \mathrm{~h}$. Cellulose acetate was saponified in $0.1 \mathrm{M}$ ethanolic 
solution of sodium hydroxide at $40^{\circ} \mathrm{C}$ for $2 \mathrm{~h}$. After saponification, the film of regenerated cellulose was thoroughly rinsed by MQ water. In order to increase film rigidity it was annealed in MQ water at $80^{\circ} \mathrm{C}$ for $5 \mathrm{~min}$ before its use in the force measurements. A piece of film of regenerated cellulose was mounted with a double-side adhesive tape onto the specimen disk of an AFM liquid cell (Park Scientific Instruments). Prior to force measurements, surfaces of cellulose films were imaged in $0.1 \mathrm{mM} \mathrm{NaCl}$ solution by AFM. For a $1 \times 1 \mu \mathrm{m}$ scan size, the RMS roughness did not usually exceed $1.0 \mathrm{~nm}$.

Colloid probes were prepared by attaching a cellulose sphere (Kohjin Co. Ltd., Japan, diameter 3-15 $\mu \mathrm{m}$ ) to the end of a v-shaped tipless cantilever using DP105 two-component epoxy adhesive (FineCal, Cardiff, UK). The tipless cantilevers were silicon ultralevers (Veeco Instruments) with a specified spring constant of $0.4 \mathrm{~N} / \mathrm{m}$. Attachment of the cellulose particles was achieved by the use of a micromanipulator (Singer Instruments Ltd.), which allowed precise location of the particle at the apex of the cantilever. Great care was taken to ensure that a minimum amount of glue was used in the attachment procedure, and that the lower surface of the sphere was not coated with glue. Spheres with diameter between 3 and $6 \mu \mathrm{m}$ were selected for probe preparation.

Both cellulose films and cellulose spheres were modified with CBD by exposure of the cellulose samples to a CBD aqueous solution with protein content of $\sim 0.1 \mathrm{mg}$ per $\mathrm{ml}$ for $30 \mathrm{~min}$. Cellulose samples treated with CBD were then rinsed with MQ water and were used for force measurements on the day of preparation.

\subsection{Force measurements}

The forces between plain cellulose surface and cellulose particle were measured using the scanning probe microscopy-colloid probe. An Autoprobe CP-100 (Veeco Instruments) was used for all force measurements in an aqueous environment using a liquid cell. The cantilever spring constant used throughout this study was $0.38( \pm 0.04)$ $\mathrm{N} \mathrm{m}^{-1}$, an averaged value of the spring constant taken from the calibration of cantilevers across the wafer used. The mass of the colloid probes used is small compared to the mass of the cantilever, and they are carefully positioned at the cantilever apex, so the specified value of the spring constant is valid throughout the study. To avoid an influence of hydrodynamic conditions and loading force on surface interactions, all force measurements were carried out at the low loading force of $6 \mathrm{nN}$ with a slow rate of scanner movement of $0.1 \mathrm{~Hz}$ and a scanner sweep range of $0.4 \mu \mathrm{m}$. At such settings, all measurements with cellulose surfaces and cellulose surfaces containing CBD gave force-distance curves covering all descriptive regions avoiding signal saturation. Zero separation was determined from the region of constant compliance that corresponds to a linear increase of deflection in response to the driving piezoscanner. The data have been represented as the average of five approach-retraction cycles in different places across the sample of cellulose film. The forces were normalised by the radius of the probe, which was determined by online video capture software linked to an optical microscope.

The force measurements were carried out in the following order on a combination of unmodified and modified cellulose surfaces with the same cellulose probe: two pure cellulose surfaces; modified cell film and pure cellulose sphere; pure cellulose sphere and modified-cellulose sphere; both modified-cellulose surfaces.

\subsection{Sample imaging}

Topography and surface roughness of cellulose films were determined from flattened, but otherwise unfiltered AFM images. Images in both liquid media and air were obtained in contact and tapping modes using Dimension 3100 scanning probe microscope (Veeco Instruments).

\subsection{Surface charge of cellulose surfaces}

Zeta potentials of cellulose particles were obtained from electrophoretic mobility of cellulose spheres in a range $\mathrm{NaCl}$ solutions varying electrolyte concentration from 0.1 to $50 \mathrm{mM}$. Measurements of electrophoretic mobility were made using a ZetaSizer 2000/3000 (Malvern Instruments). Zeta potentials were calculated using software provided by instrument manufacture.

Zeta potentials of cellulose film were obtained from streaming potential measurements implemented with the use of EKA Electro Kinetic Analyzer (Anton Paar GmbH, Austria) in $0.1-50 \mathrm{mM} \mathrm{NaCl}$ solutions. Zeta potentials were calculated using software provided by producer of the analyzer.

These procedures were used for characterization of surface charge for both pure cellulosic materials and modified by CBD.

\section{Results and discussion}

\subsection{Interaction of the surfaces of pure cellulose}

Fig. 1 shows typical measurements for the interaction of two cellulose surfaces. The approach curves are repulsive in electrolyte solutions of any ionic strength, whereas retraction curves showed very small adhesion at small separations in the solution of the highest ionic strength. Hysteresis is observed between the curves measured upon separation and approach at all ionic strength of electrolyte solution. The force-distance curves are dependent on the electrolyte concentration. Analysis of electrolyte-induced changes in force-distance profiles allows discrimination of the following susceptible features that characterize the changes in the interaction (Fig. 1c): value of distance for the onset of the 

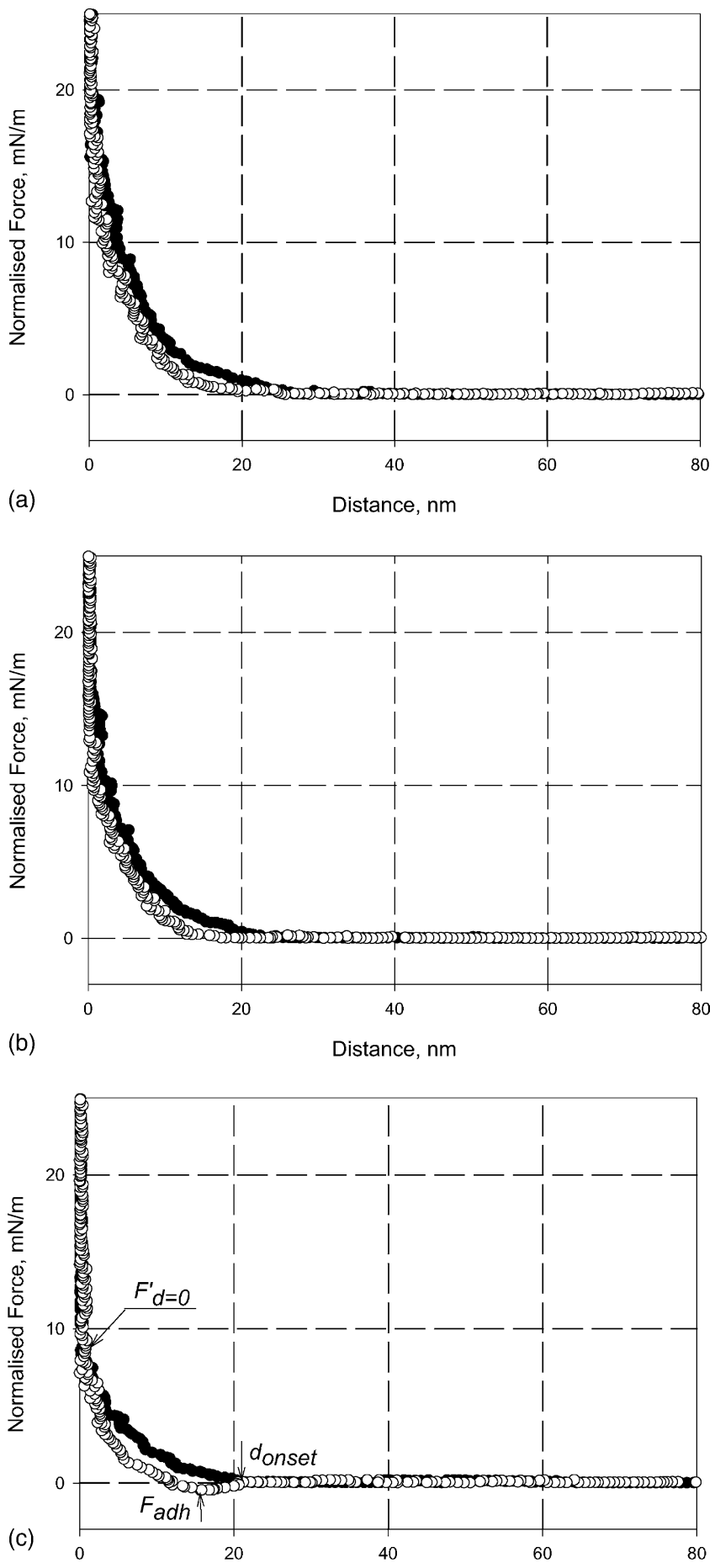

Fig. 1. Force-distance profiles for the interaction of two surfaces of pure cellulose at different ionic strength: (a) $0.1 \mathrm{mM} \mathrm{NaCl}$; (b) $1 \mathrm{mM} \mathrm{NaCl}$; (c) $10 \mathrm{mM} \mathrm{NaCl}$. (O) Approach curves; $(\bigcirc)$ retraction curves.

repulsive forces $\left(d_{\text {onset }}\right)$, value of adhesion force $\left(F_{\mathrm{adh}}\right)$ and magnitudes of resulting force at the beginning of constant compliance $\left(F_{d=0}^{\prime}\right)$. Due to smooth gradual changes in obtained force profiles, the last characteristic is useful for comparative purposes: the lower $F_{d=0}^{\prime}$ values mean lower magnitude of net force through the entire force sweep. To
Table 1

Characteristic features of force profiles for the interaction of the surfaces of pure cellulose at different ionic strength

\begin{tabular}{lllc}
\hline Ionic strength $(\mathrm{mM})$ & $d_{\text {onset }}(\mathrm{nm})$ & $F_{d=0}^{\prime}(\mathrm{mN} / \mathrm{m})$ & $F_{\text {adh }}(\mathrm{mN} / \mathrm{m})$ \\
\hline 0.1 & $\frac{25.3 \pm 0.8}{24.5 \pm 0.7}$ & $\frac{19.6 \pm 1.1}{16.8 \pm 0.7}$ & $\frac{\mathrm{N} / \mathrm{A}}{\mathrm{N} / \mathrm{A}}$ \\
\multirow{2}{*}{1} & $\frac{22.4 \pm 0.7}{16.4 \pm 0.8}$ & $\frac{16.1 \pm 0.8}{12.8 \pm 0.6}$ & $\frac{\mathrm{N} / \mathrm{A}}{\mathrm{N} / \mathrm{A}}$ \\
\multirow{2}{*}{10} & $\frac{20.2 \pm 0.8}{\mathrm{~N} / \mathrm{A}}$ & $\frac{10.8 \pm 0.7}{9.5 \pm 0.6}$ & $\frac{\mathrm{N} / \mathrm{A}}{0.48 \pm 0.06}$ \\
\hline
\end{tabular}

Upper figures correspond to approach curves, lower ones correspond to retraction curves. N/A, not applicable: the feature is not discernable. $F_{\text {adh }}$ is not determinable for completely repulsive profiles. In case of adhesion on separation curves $d_{\text {onset }}$ was not determined.

avoid discussion of crowded illustrations with overlapping data points, the characteristics of the interaction in the terms of these features are presented and only representative force curves and important data are shown.

The force-distance profiles obtained in this study are generally consistent with AFM or SFA studies of the cellulose/cellulose interaction [4-8]. The influence of electrolyte concentration on the force-distance profiles signifies the importance of the electrostatic interactions for cellulose surfaces (Fig. 1, Table 1). Negative surface charge on both cellulose film and cellulose sphere was confirmed by electrokinetic measurements (Table 2). Both cellulose materials were produced through alkaline regeneration of cellulose derivatives which appears to cause an oxidative degradation of cellulose chains resulting in carboxylic groups in molecular structure of the regenerated cellulose. Dissociation of these groups is responsible for negative surface charge and, consequently, for repulsive interaction of the cellulose surfaces. It is generally agreed $[5,7,8]$ that interactions between two cellulose surfaces at relatively large separation distances can be described using DVLO theory. However, for interactions between polymeric surfaces one must consider electrosteric forces that arise due to interactions of thermally mobile polymeric chains or segments that protrude from the surface into the medium. The extent of chain protuberance and, consequently, range of electrosteric forces depends

Table 2

$\xi$-Potential $(\mathrm{mV})$ of cellulose sphere and film before (upper figures) and after (lower figures) modification with CBD at different ionic strength of background electrolyte

\begin{tabular}{|c|c|c|}
\hline Ionic strength (mM) & Cellulose sphere & Cellulose flat surface \\
\hline \multirow{2}{*}{0.1} & $-35.6 \pm 1.0$ & $-11.4 \pm 0.9$ \\
\hline & $\overline{-37.3 \pm 1.8}$ & $\overline{-12.3 \pm 0.8}$ \\
\hline \multirow{2}{*}{1} & $-30.8 \pm 2.0$ & $-10.2 \pm 1.3$ \\
\hline & $\overline{-32.5 \pm 1.5}$ & $\overline{-11.5 \pm 1.1}$ \\
\hline \multirow{2}{*}{2} & $-29.3 \pm 1.2$ & $-7.4 \pm 1.0$ \\
\hline & $\overline{-31.0 \pm 0.9}$ & $\overline{-8.6 \pm 1.1}$ \\
\hline \multirow{2}{*}{10} & $-18.2 \pm 2.6$ & $-6.1 \pm 1.2$ \\
\hline & $\overline{-21.8 \pm 1.7}$ & $\overline{-7.2 \pm 0.9}$ \\
\hline \multirow{2}{*}{20} & $\underline{-14.1 \pm 1.1}$ & $-3.8 \pm 0.9$ \\
\hline & $\overline{-17.0 \pm 0.9}$ & $\overline{-4.5 \pm 1.2}$ \\
\hline
\end{tabular}


on surface roughness and polymer/solvent interactions. Because chains of ionisable polymer are involved in such interaction these forces are referred to electrosteric forces [5].

The force profile measured by AFM is a combination of various attractive and repulsive forces exerted between two surfaces. For relatively large distances (typically larger than $10 \mathrm{~nm}$ ) the only long range attractive force, which could compete with the double layer and electrosteric forces, are hydrophobic interactions. Significant hydrophobic forces are unlikely to develop on the highly hydrophilic cellulose surfaces.

Like electrostatic forces electrosteric interactions also depend on the ionic strengths of the solution. The dominant forces therefore are rather difficult to discriminate if both forces are acting between the surfaces. As can be seen from Table 2, negative surface charge for both cellulose materials decreases with increase in ionic strength. Therefore, decreased values of repulsive force onset and normalised force magnitude with increase of ionic strength (Fig. 1, Table 1) are consistent with the concept of both long range double layer forces and electrosteric forces arising between two charged surfaces in aqueous solutions. Therefore, it is safe to assume that the forces governing the interaction between the surfaces of pure cellulose at relatively large separation distances are dominated by double layer and steric factors.

The situation is much more complicated for the force profile at smaller distances. The force within this range between two surfaces is an interplay of the forces of double layer, electrosteric, van der Waals, solvation, and hydration interactions. The combination of the forces mentioned above could result in both repulsive and attractive force at small separation distances. It is worth noting that retraction curves are acquired after the contact of two interacting surfaces which enables formation of physical inter-surface binding. Hydrogen bonding seems a likely outcome of the contact between two cellulose surfaces. Physical contact also increases the relative strength of short-range attractive van der Waals forces. These factors may result in less repulsive force profiles compared with curves obtained on surface approach. As a matter of fact, hysteresis between approach and retraction curves observed at any ionic strength suggests that the interaction between the surfaces of pure cellulose become less repulsive after surface contact. Furthermore, at higher ionic strength $(10 \mathrm{mM}$, Fig. 1b) retraction force curves show a slight adhesive force. Decrease of surface charge with increased electrolyte concentration diminishes repulsive forces enabling adhesion on retraction of cellulose surfaces at higher ionic strength.

It should be noted that despite the generally good agreement of our force profiles with those obtained by other authors, there are some quantitative discrepancies between both earlier published results and results presented in this paper. First of all, in the range of the interaction, the onset of repulsive forces according to our observation never exceeds $25 \mathrm{~nm}$. This corresponds to results reported by Rutland and co-workers $[4,5]$ in studies of the interaction between two cellulose spheres. Whereas in measurements carried out by Zauscher and Klinberg using a cellulose film and sphere [8] the onset of repulsive forces reached almost $100 \mathrm{~nm}$ at low ionic strength $(0.1 \mathrm{mM} \mathrm{NaCl})$. The differences in samples of cellulose materials used could explain these discrepancies. We used a cellulose film with very smooth surfaces. Moreover, thermal annealing of a cellulose film increases its rigidity and, as a result, decreases viscoelastic deformation of cellulose material under pressure of contacting surfaces. Sample deformation could result in erroneous determination of zero-distance during the conversion of raw data into force as a function of sample-probe separation. Taking into consideration that cellulose is more swollen and more deformable at lower ionic strength, such deformation could especially affect results at lower ionic strength.

\subsection{Interaction of the surfaces of cellulose modified by $C B D$}

Surface morphology and surface charge are important factors in interfacial interactions, thus the influence of cellulose modification by CBD on these properties was determined. Fig. 2 demonstrates that in the chosen modification conditions CBDs distribute across the cellulose flat surface as agglomerates of different sizes, with maximal size up to $80 \mathrm{~nm}$ causing an increase in the RMS roughness of the cellulose surface from less than 0.4 to $2.6 \pm 0.3 \mathrm{~nm}$. CBDs are characterised by hydrophobic properties $[19,28]$ and CBDs molecules tend to aggregate in aqueous solution [28] due to hydrophobic interactions. As a result, CBDs are likely to adsorb by cellulose surface as agglomerates rather than single molecules. Moreover, as for any specific binding, there is a need for specific spatial pre-orientating of CBD over the cellulose surface. With some CBDs already deposited on the surface pre-orientation could be obstructed causing preferential CBD binding to adsorbed CBD rather than to the free patches of cellulose surface. This would lead to building up of CBD deposits and also contribute to the formation of the CBD agglomerates on the surface. Such an adsorption mechanism will result in increase of the surfaces roughness.

Cellulose modification by CBDs causes an increase in negative charge of cellulose surface (Table 2). This suggests that adhesive forces due to interaction of the opposite charged surfaces could be more susceptible to charge variation. To confirm this the interaction of a cellulose spheres with and without CBD coating to a positively charged surface covered with chitosan was measured. It can be seen from Fig. 3, that the cellulose/chitosan force curve shows clear adhesion on retraction of the surfaces demonstrating that CBD adsorption was able to cause changes in double layer and electrosteric forces detectable by AFM with single cellulose sphere. Modification of cellulose spheres with CBD increases both the magnitude and range of adhesion on retraction, while approach curves remain repulsive. Thus, alterations in double layer and electrosteric forces caused by cellulose modification with CBD are detectable by AFM. 

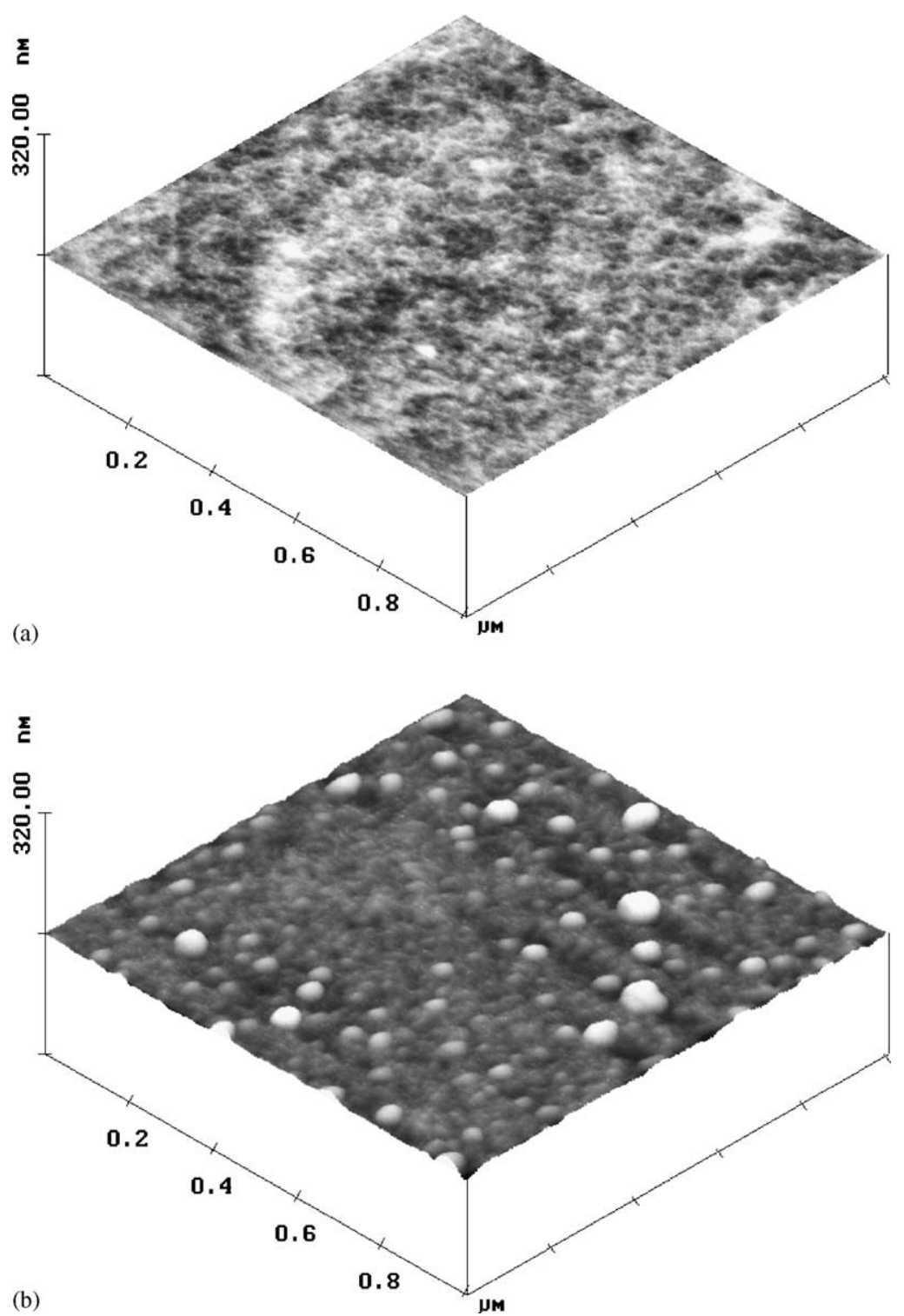

Fig. 2. AFM images of a cellulose film (a) and a cellulose film modified with CBD (b) obtained in the air.

When interpreting the increase in adhesion of CBD-modified sphere to a chitosan surface, one should consider the possibility of a contribution by specific binding to chitosan. It is known [26] that some CBDs of family I also have an affinity for chitin.

As can be seen from Fig. 4, general shape of the force profiles retained in the measurements involving cellulose surfaces modified with CBD. For both two types of CBDs used and different combinations of modified and unmodified cellulose materials (modified film/unmodified sphere, unmodified film/modified sphere, two modified surfaces) approach curves are repulsive and retraction curves show slight adhesion especially at higher ionic strength (e.g. $10 \mathrm{mM}$ ). However, cellulose modification with CBDs results in discernable changes of the characteristic features of the force profiles.

According to Tables 1, 3 and 4, cellulose modification significantly decreases the distance of the onset of repulsive force $\left(d_{\text {onset }}\right)$ for both studied CBDs. This characteristic remains dependent on the ionic strength. It decreases with the increase of the electrolyte concentration. This suggests that in the case of the interaction of cellulose surfaces modified with CBDs double layer repulsive forces also prevail. However, increase of the negative $\xi$-potential of cellulose surface due to CBD binding should increase both magnitude and range of the repulsive force. On the contrary, both $d_{\text {onset }}$ and $F^{\prime}{ }_{d=0}$ decrease for the interaction of cellulose surfaces that involves at least one surface modified by CBDs. The net interaction forces are less repulsive when compared with the interaction of pure cellulose surfaces. To explain this discrepancy one should consider the topography of the material with surface bound protein and the forces controlling protein interactions.

Determination of surface $\xi$-potential is based on electrokinetic phenomena via measurements of a net characteristic as 

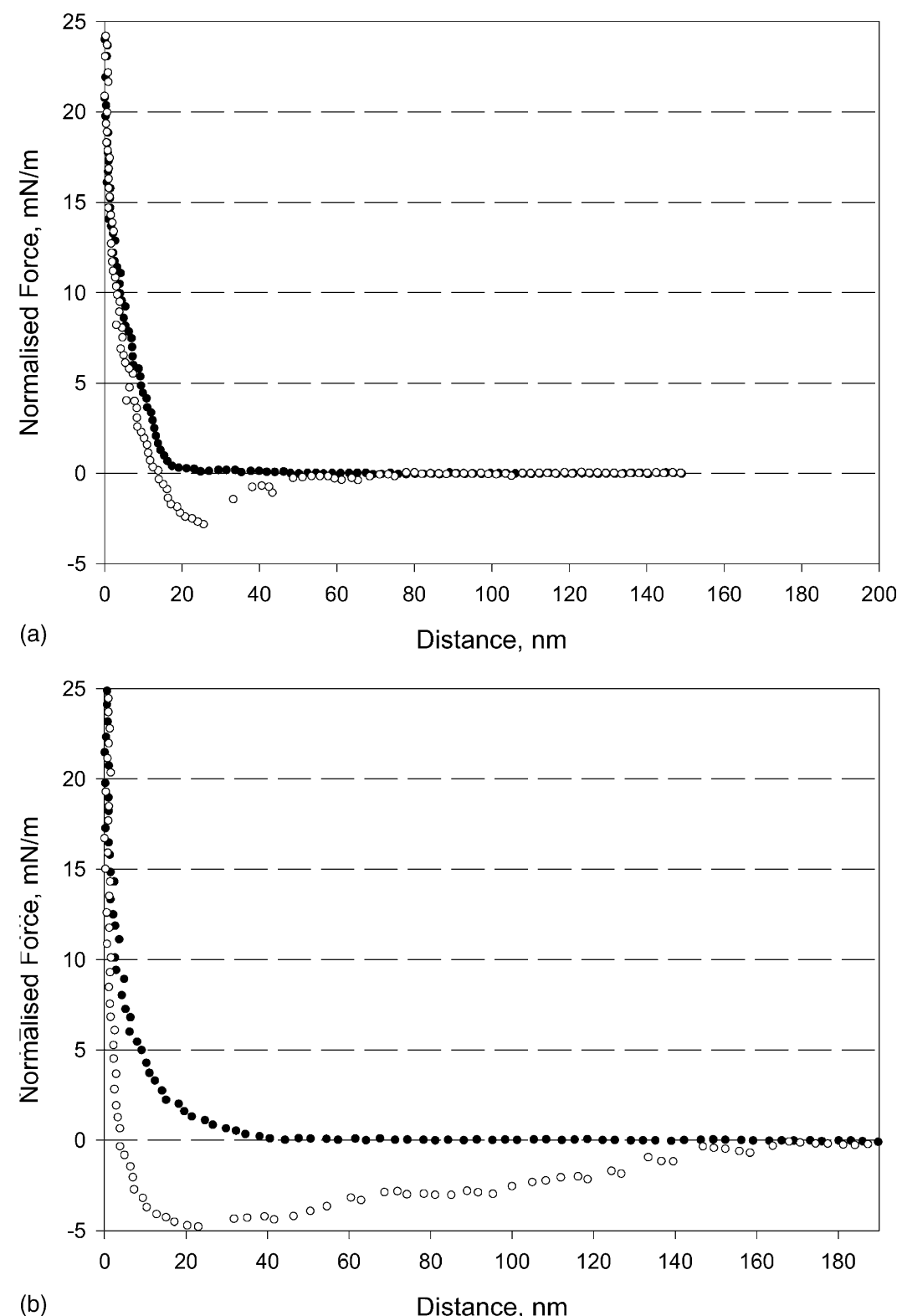

Fig. 3. Force-distance profiles for the interaction of a pure cellulose sphere (a) and a cellulose sphere modified with CBD (b) with a cellulose film covered by chitosan in $1 \mathrm{mM} \mathrm{NaCl}$. (O) Approach curves; $(\bigcirc)$ retraction curves.

electrophoretic mobility in case of sphere, or streaming potential in case of flat surface, assuming even distribution of the charge across the surface. However, distinctive properties of protein surfaces, and consequently surface of materials with bound proteins, are irregular topography of protein surface and non-uniform distribution of surface charges. CBDs of family I fold into a small wedge shaped structure formed by a small irregular triple stranded $\beta$-sheet [13]. One face of the wedge contains a row of three tyrosines together with a few potential hydrogen bond forming residues. It is obvious that such a structure is characterised by a variety of surface functional groups with irregular distribution on the protein surface. It is generally agreed that the surface of the wedge, containing tyrosine residues, is responsible for the specific binding to cellulose. This results in specific orientation of CBDs at monomolecular sorption. However, agglomeration of CBD adsorbed on the cellulose surface leads to diversity in interfacial orientation of CBDs. This includes orientation of tyrosine containing row against the supporting cellulose surface. It is worth noting that for the HFBI-DCBD, which is a double CBD, orientation of the CBD segment involved in specific binding against the cellulose support is possible even at monomolecular adsorption. Thus, despite an increase in the net negative charge of the cellulose surface modified with CBDs, formation of patches with positive surface charge is quite conceivable.

It is generally recognised that non-uniform electrostatic fields guide intermolecular binding trajectories [29]. Long 

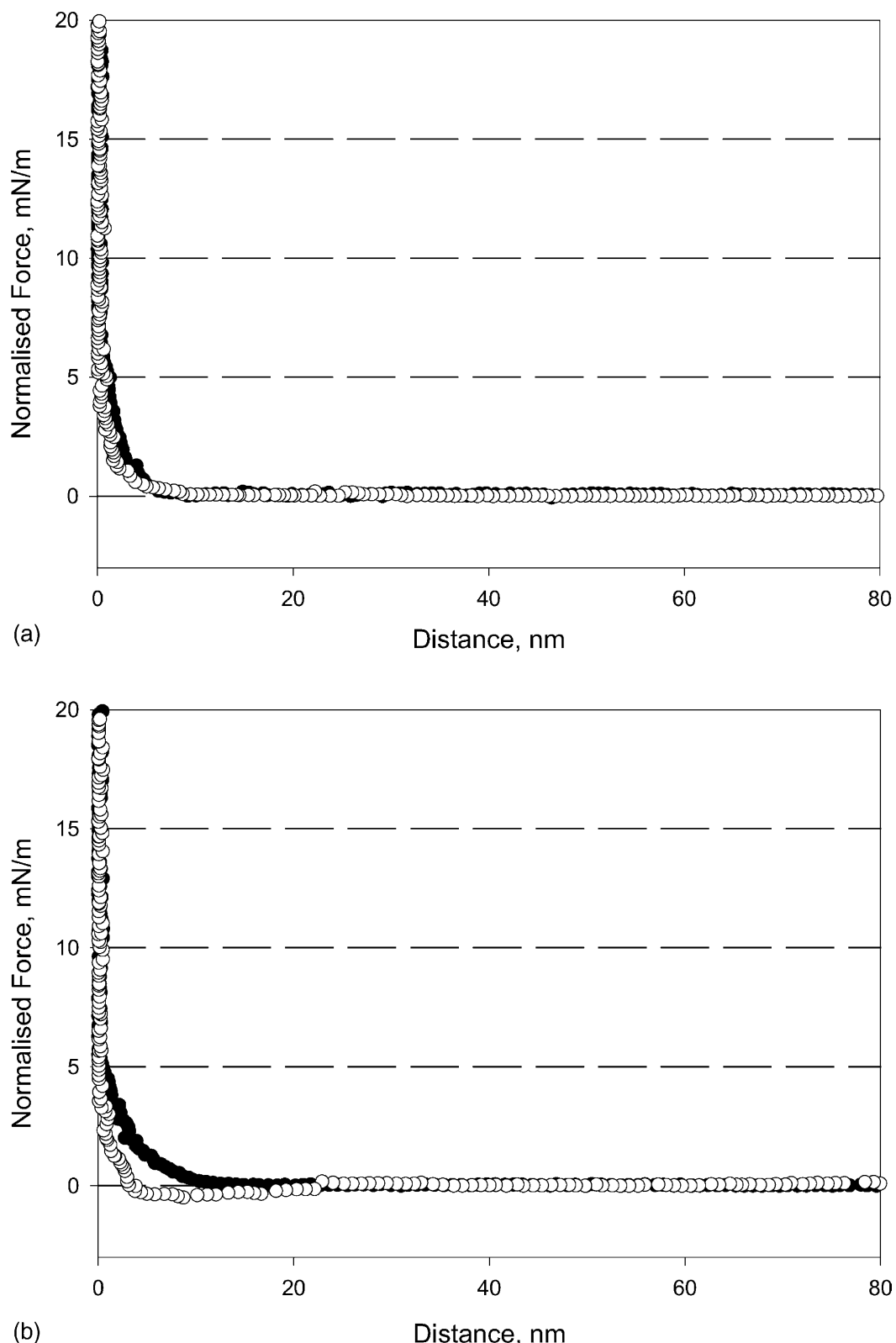

Fig. 4. Force-distance profiles for the interaction of a cellulose flat surface with a cellulose sphere modified by CBD (a) and HFBI-DCBD (b) in $10 \mathrm{mM}$ $\mathrm{NaCl}$. (O) Approach curves; $(\bigcirc)$ retraction curves.

range electrostatic attractive forces steer changes in molecular conformation to overcame the repulsive forces between similarly charged molecules and provide spatial orientation of ligand groups and binding sites. Double layer attractive forces between negatively charged surface or patches of surface with bound CBD and positively charged patches of the CBD arise at long distances of surface separation. These compensate for double layer repulsive forces resulting in less repulsive force profiles with decreased range and magnitude of net repulsive force. Hydrophobic forces caused by CBD binding on the surfaces competing with double layer force at large separation distances could reduce the measured repulsive force. However, the interaction involves highly hydrophilic cellulose which minimises such a hydrophobic contribution.

Absence of attractive interaction on approach curves at any ionic strength indirectly confirms this assumption. Increase of ionic strength suppresses the double layer repulsive force. This could lead to the dominance of attractive forces provided they do not depend on the electrolyte concentration. On approach an attractive interaction is absent even at higher ionic strength proving that both repulsive and attractive force are mainly double layer forces.

Cellulose modification with CBD significantly decreases the net repulsive force before surfaces come into contact $\left(F^{\prime} d=0\right.$ on approach curves, Tables 1, 3 and 4). Thus, CBD 
Table 3

Characteristic features of force profiles for the interaction of the cellulose surfaces modified with CBD at different ionic strength

\begin{tabular}{|c|c|c|c|}
\hline $\begin{array}{l}\text { Ionic strength } \\
(\mathrm{mM})\end{array}$ & $d_{\text {onset }}(\mathrm{nm})$ & $F_{d=0}^{\prime}(\mathrm{mN} / \mathrm{m})$ & $F_{\text {adh }}(\mathrm{mN} / \mathrm{m})$ \\
\hline \multicolumn{4}{|c|}{ Cellulose film interacting with cellulose sphere modified by CBD } \\
\hline \multirow{2}{*}{0.1} & $20.0 \pm 0.8$ & $11.4 \pm 0.9$ & $\mathrm{~N} / \mathrm{A}$ \\
\hline & $\overline{15.4 \pm 0.6}$ & $\overline{7.6 \pm 0.8}$ & $\overline{\mathrm{N} / \mathrm{A}}$ \\
\hline \multirow{2}{*}{1} & $15.1 \pm 0.6$ & $7.7 \pm 0.8$ & $\mathrm{~N} / \mathrm{A}$ \\
\hline & $\overline{14.8 \pm 0.5}$ & $\overline{6.9 \pm 0.7}$ & $\overline{\mathrm{N} / \mathrm{A}}$ \\
\hline \multirow{2}{*}{10} & $7.8 \pm 0.5$ & $\underline{5.1 \pm 0.6}$ & $\mathrm{~N} / \mathrm{A}$ \\
\hline & $\overline{6.3 \pm 0.5}$ & $\overline{5.6 \pm 0.6}$ & $\overline{\mathrm{N} / \mathrm{A}}$ \\
\hline \multicolumn{4}{|c|}{ Cellulose film modified by CBD interacting with cellulose sphere } \\
\hline \multirow{2}{*}{0.1} & $20.5 \pm 0.7$ & $15.5 \pm 0.7$ & $\mathrm{~N} / \mathrm{A}$ \\
\hline & $\overline{19.5 \pm 0.6}$ & $\overline{12.3 \pm 0.8}$ & $\overline{\mathrm{N} / \mathrm{A}}$ \\
\hline \multirow{2}{*}{1} & $17.9 \pm 0.5$ & $11.1 \pm 0.6$ & $\mathrm{~N} / \mathrm{A}$ \\
\hline & $\overline{16.1 \pm 0.5}$ & $\overline{10.0 \pm 0.6}$ & $\overline{\mathrm{N} / \mathrm{A}}$ \\
\hline \multirow{2}{*}{10} & $14.0 \pm 0.6$ & $8.5 \pm 0.5$ & $\mathrm{~N} / \mathrm{A}$ \\
\hline & $\overline{10.6 \pm 0.5}$ & $\overline{8.8 \pm 0.6}$ & $\overline{\mathrm{N} / \mathrm{A}}$ \\
\hline \multicolumn{4}{|c|}{ Interacting of cellulose surfaces both modified by $\mathrm{CBD}$} \\
\hline \multirow{2}{*}{0.1} & $19.5 \pm 0.8$ & $13.2 \pm 0.8$ & $\mathrm{~N} / \mathrm{A}$ \\
\hline & $8.5 \pm 1.0$ & $\overline{12.1 \pm 0.9}$ & $\overline{\mathrm{N} / \mathrm{A}}$ \\
\hline \multirow{2}{*}{1} & $17.7 \pm 0.7$ & $12.9 \pm 0.6$ & $\mathrm{~N} / \mathrm{A}$ \\
\hline & $\overline{15.8 \pm 0.5}$ & $\overline{12.8 \pm 0.5}$ & $\overline{\mathrm{N} / \mathrm{A}}$ \\
\hline \multirow{2}{*}{10} & $16.3 \pm 0.6$ & $12.3 \pm 0.5$ & $\mathrm{~N} / \mathrm{A}$ \\
\hline & $\mathrm{N} / \mathrm{A}$ & $\overline{11.9 \pm 0.5}$ & $\overline{0.26 \pm 0.05}$ \\
\hline
\end{tabular}

Upper figures correspond to approach curves, lower ones correspond to retraction curves.

Table 4

Characteristic features of force profiles for the interaction of the cellulose surfaces modified with HFBI-DCBD at different ionic strength

\begin{tabular}{|c|c|c|c|}
\hline $\begin{array}{l}\text { Ionic strength } \\
(\mathrm{mM})\end{array}$ & $d_{\text {onset }}(\mathrm{nm})$ & $F_{d=0}^{\prime}(\mathrm{mN} / \mathrm{m})$ & $F_{\text {adh }}(\mathrm{mN} / \mathrm{m})$ \\
\hline \multicolumn{4}{|c|}{ Cellulose film interacting with cellulose sphere modified by CBD } \\
\hline \multirow{2}{*}{0.1} & $20.2 \pm 0.6$ & $17.1 \pm 0.9$ & $\mathrm{~N} / \mathrm{A}$ \\
\hline & $\overline{13.6 \pm 0.5}$ & $\overline{7.7 \pm 0.6}$ & $\overline{\mathrm{N} / \mathrm{A}}$ \\
\hline \multirow{2}{*}{1} & $19.6 \pm 0.5$ & $11.6 \pm 0.9$ & $\mathrm{~N} / \mathrm{A}$ \\
\hline & $\mathrm{N} / \mathrm{A}$ & $\overline{3.1 \pm 0.4}$ & $\overline{0.28 \pm 0.05}$ \\
\hline \multirow{2}{*}{10} & $9.6 \pm 0.4$ & $4.8 \pm 0.4$ & $\mathrm{~N} / \mathrm{A}$ \\
\hline & $\overline{N / A}$ & $\overline{2.9 \pm 0.5}$ & $0.50 \pm 0.06$ \\
\hline \multicolumn{4}{|c|}{ Cellulose film modified by CBD interacting with cellulose sphere } \\
\hline \multirow{2}{*}{0.1} & $13.8 \pm 0.5$ & $15.6 \pm 0.8$ & $\mathrm{~N} / \mathrm{A}$ \\
\hline & $\mathrm{N} / \mathrm{A}$ & $\overline{13.4 \pm 0.6}$ & $\overline{0.69 \pm 0.08}$ \\
\hline \multirow{2}{*}{1} & $12.2 \pm 0.5$ & $14.2 \pm 0.7$ & $\mathrm{~N} / \mathrm{A}$ \\
\hline & $\mathrm{N} / \mathrm{A}$ & $\overline{10.2 \pm 0.5}$ & $\overline{0.91 \pm 0.10}$ \\
\hline \multirow{2}{*}{10} & $6.8 \pm 0.4$ & $5.5 \pm 0.3$ & $\mathrm{~N} / \mathrm{A}$ \\
\hline & $\mathrm{N} / \mathrm{A}$ & $\overline{3.5 \pm 0.3}$ & $\overline{1.05 \pm 0.10}$ \\
\hline \multicolumn{4}{|c|}{ Interacting of cellulose surfaces both modified by $\mathrm{CBD}$} \\
\hline \multirow{2}{*}{0.1} & $18.7 \pm 0.6$ & $10.3 \pm 0.7$ & $\mathrm{~N} / \mathrm{A}$ \\
\hline & $\mathrm{N} / \mathrm{A}$ & $\overline{7.4 \pm 0.4}$ & $\overline{0.14 \pm 0.2}$ \\
\hline \multirow{2}{*}{1} & $17.4 \pm 0.6$ & $7.3 \pm 0.6$ & $\mathrm{~N} / \mathrm{A}$ \\
\hline & $\mathrm{N} / \mathrm{A}$ & $\overline{5.6 \pm 0.5}$ & $\overline{0.14 \pm 0.3}$ \\
\hline \multirow{2}{*}{10} & $8.3 \pm 0.5$ & $5.8 \pm 0.4$ & $\mathrm{~N} / \mathrm{A}$ \\
\hline & $\mathrm{N} / \mathrm{A}$ & $\overline{4.3 \pm 0.4}$ & $\overline{0.77 \pm 0.07}$ \\
\hline
\end{tabular}

Upper figures correspond to approach curves, lower ones correspond to separation curves. binding to the cellulose surface facilitates interfacial contact of two cellulose surfaces if at least one surface is modified with CBD. At small separation distances, attractive van der Waals forces contribute to reducing the net repulsive force enhancing contact and inter-molecular binding. Comparison of the characteristic features of force profiles on approach (Tables 1, 3 and 4) confirms an enhancement of inter-surface interactions due to CBD binding to cellulose surfaces. Profiles obtained in measurements with, at least, one modified cellulose surface are characterised by a lower repulsive net force and force range. However, as a result of cellulose modification with the single CBD, adhesion between surfaces does not practically occur despite lowering of the repulsive net force. Only for interaction of two cellulose surfaces modified by the single CBD a lower adhesion is observed at high ionic strength. On the contrary, binding HFBI-DCBD to cellulose surfaces causes an adhesion force even at low electrolyte concentration. This can be explained by the difference in the structure of these CBDs. HFBI-DCBD consists of two CBDs joined together by a linker that has the possibility of binding CBD fragments to different interacting surfaces. Due to CBD aggregation the availability of free cellulose binding sites on the surface of cellulose modified by single CBD could be as high as the availability in the case of HFBI-DCBD. This promotes inter-surface attractive force at approach of the surfaces modified by CBDs of both type. However, at separation the difference in CBDs molecular structure alters adhesion between surfaces. HFBI-DCBDs are able to cross-link two interacting surfaces with binding domains adsorbed on the different surfaces. In case of such binding, surface separation leads to breaking of highly stable specific bonds between CBD and cellulose. On the contrary, for the surfaces modified with single CBD during separation weaker protein/protein inter-molecular bonds are disrupted. It is interesting that for cellulose with adsorbed single CBD adhesion forces are even weaker than in the case of the interaction of pure cellulose surfaces $(0.26 \mathrm{mN} / \mathrm{m}$ against $0.48 \mathrm{mN} / \mathrm{m}$ at ionic strength of $10 \mathrm{mM}$ ). Hydrogen bonding between cellulose surfaces, which arises after contact of two pure cellulose surfaces, is obviously stronger than protein/protein interactions.

Adhesive forces in the interaction of cellulose surfaces modified with HFBI-DCBD increases with electrolyte concentration (Table 4). The involvement of $\mathrm{H}$-bonds in binding of CBD to cellulose is widely accepted $[13,14]$. With increase of ionic strength these bonds are increasingly dominant while the repulsive double layer forces are decreasing. Adhesive forces tend to be lower when both surfaces are modified with HFBI-DCBD compared to the adhesion between modified/unmodified cellulose surfaces (Table 4). Covering both cellulose surfaces with HFBI-DCBD decreases accessibility of cellulose patches for further binding HFBI-DCBD. This reduces number of specific binding events and, consequently, adhesion force at separation. However, this does not affect attractive forces during surface approaches because of involvement of long-range double 
layer forces. As a result, force profiles obtained, where both surfaces are modified with HFBI-DCBD, are the least repulsive, but with lower adhesion forces.

Although measurements carried out with different combinations of modified and unmodified surfaces give clear evidence of reinforcing of attractive forces due to CBD binding, there are quantitative differences which depend on the use of a modified film or sphere. This could be related to non-uniformity in distribution of CBDs adsorbed on the surface. This is especially important for a sphere. Sphere modifications with CBD could result in a different coverage of the most crucial, small area of cellulose sphere which contacts with the cellulose film in force measurements. This could happen for spheres modified by CBDs of different types. Moreover, cellulose films and spheres are characterised by different supramolecular and morphological structure (e.g. crystallinity, surface topography) influencing CBD binding. This could give discrepancies in comparison of results when using a modified sphere or a film.

Evidence for increases in the attractive forces due to modification of cellulose surfaces obtained from the AFM measurements can be used to explain the results observed in the application of cellulose fibres modified with CBD. Drainage rate of pulp of CBD-modified cellulose decreases compared with that of unmodified cellulose fibres [18]. This is consistent with the reduction of inter-surface repulsion, which results in fibre agglomeration and reduction of the drainability. Modification of cellulose fibres from CBD solutions of low concentration improves the mechanical strength of paper produced from the modified fibres due to increased attractive forces. However, increased levels of bound CBD reduce paper mechanical properties because CBDs decrease the accessibility of the pure cellulose patches. An improvement of paper strength as a result of treatment with double CBD observed by previous research [19] is in complete agreement with the appearance of adhesion force between the cellulose surfaces treated with HFBI-DCBD.

\section{Conclusion}

Colloidal probe microscopy has been used to study the interactions between model cellulose surfaces and the influence of CBD, peptides specifically bound to cellulose, on interfacial interaction of cellulose surfaces modified with CBDs.

The interaction between pure cellulose surfaces in aqueous electrolyte solution is dominated by double layer repulsive forces with the range and magnitude of net force dependable on electrolyte concentration. Such interaction is attributed to the negative charge of cellulose surfaces. Despite an increase in surface charge owing to CBD binding to the cellulose surface, force profiles are less repulsive for interactions involving, at least, one modified surface. Irregular topography of protein surface and non-uniform distribution of surface charges causes irregular electrostatic fields on the surface of modified cellulose. This non-uniformity arises even in the case of monomolecular CBD adsorption. In fact, morphological studies reveal agglomeration of CBD adsorbed on a cellulose surface. Such a structure results in a variety of surface functional groups that are irregularly distributed across modified cellulose surface.

Non-uniform electrostatic fields are recognised as source of forces guiding intermolecular binding trajectories. Longrange electrostatic attractive forces generate inter-molecular steering torques to surmount repulsive forces between similarly charged surfaces and provide spatial orientation of ligand groups and binding sites. It is supposed that positively charged patches exist on the surface of cellulose modified with CBDs. Double layer attractive forces within these regions compensate for double layer repulsive forces resulting in less repulsive force profiles with a decrease in both range and magnitude of the net repulsive force.

Both single and double CBD decrease the net repulsive force between cellulose surfaces. However, binding HFBI-DCBD to cellulose surfaces causes adhesion force at separation, whereas retraction force curves obtained with cellulose modified with single CBD show small adhesion only at high ionic strength. The presence of free cellulose binding sites on the surface of cellulose modified by CBD promotes inter-surface attractive forces at approach of the surfaces modified by CBDs of both types. After surface contact, these sites are able to specifically bind to touching cellulose surfaces. This potentially forms cross-links between cellulose surfaces in case of HFBI-DCBD. As a result, surface separation leads to breaking of highly stable specific bonds between CBD and cellulose in case of double CBD. In comparison, the surfaces modified with a single CBD during retraction show very small adhesion due to disruption of weaker protein/protein inter-molecular bounds. For cellulose modified with single CBD adhesion forces are even weaker than in the case of interaction of pure cellulose surfaces. Hydrogen binding between cellulose surfaces, which arises after contact of two pure cellulose surfaces, is obviously stronger than protein/protein interactions.

Covering both cellulose surfaces with HFBI-DCBD decreases adhesive forces at separation. Steric restriction lowers accessibility of cellulose patches for free binding sites of absorbed HFBI-DCBD reducing the number of specific binding events and, consequently, adhesion between the two modified surfaces.

\section{Acknowledgements}

The authors gratefully acknowledge financial support by the European Commission through the Specific Programme for research, technological development and demonstration on "Quality of life and management of living resources" of the Fifth Framework Programme. 


\section{References}

[1] J.C. Fröberg, O.J. Rojas, P.M. Claesson, Int. J. Miner. Process 56 (1999) 1.

[2] B. Capella, G. Dietler, Surf. Sci. Rep. 34 (1999) 1.

[3] C.S. Hodges, Adv. Colloid Interface Sci. 16 (2002) 13.

[4] M.W. Rutland, A. Carambassis, G.A. Willing, R.D. Neuman, Colloids Surf. A, Physicochem. Eng. Aspects 123/124 (1997) 369.

[5] A. Carambassis, M.W. Rutland, Langmuir 15 (1999) 5584.

[6] M. Holmberg, J. Berg, S. Stemme, L. Ödberg, J. Rasmusson, P. Claesson, J. Colloid Interface Sci. 186 (1997) 369.

[7] M. Österberg, P.M. Claesson, J. Adhes. Sci. Technol. 14 (2000) 603.

[8] S. Zauscher, D.J. Klingenberg, J. Colloid Interface Sci. 229 (2000) 497.

[9] S. Zauscher, D.J. Klingenberg, Colloids Surf. A, Physicochem. Eng. Aspects 178 (2001) 213.

[10] J. Urquhart, J. Intern. Med. 248 (2000) 357.

[11] P. Gemeiner, M. Polakovič, D. Mislovičová, V. Štefuca, J. Chromatogr. B, Biomed. Sci. Appl. 715 (1998) 245.

[12] O. Pajulo, J. Viljanto, B. Lönnberg, T. Hurme, K. Lönnqvist, P. Saukko, J. Biomed. Mater. Res. 32 (1996) 439.

[13] M. Linder, T. Teeri, J. Biotechnol. 57 (1997) 15.

[14] I. Levy, O. Shoseyov, Biotechnol. Adv. 20 (2002) 191.

[15] N. Din, R.N. Gilkes, B. Tekant, R.C. Miler, R.A.J. Warren, D.G. Kilburn, Biotechnology 9 (1991) 1096.

[16] R.R. Banka, S. Mishra, T.K. Ghose, World J. Microbiol. Biotechnol. 14 (1998) 551.
[17] P.J. Gao, G.J. Chen, T.H. Wang, Y.S. Zhang, J. Liu, Acta Biochim. Biophys. 33 (2001) 13

[18] H. Pala, M.A. Lemos, M. Mota, F.M. Gama, Enz. Microb. Technol. 29 (2001) 274.

[19] I. Levy, A. Nussinovitch, E. Shpigel, O. Shoseyov, Cellulose 9 (2002) 91.

[20] A. Cavaco-Paulo, J. Morgado, J. Andreaus, D. Kilburn, Enz. Microb. Technol. 25 (1999) 639.

[21] A.A. Wang, A. Mulchandani, W. Chen, Biotechnol. Prog. 17 (2001) 407.

[22] C. Kauffmann, O. Shoseyov, E. Shpigel, E.A. Bayer, R. Lamed, Yu. Shoham, R.T. Mandelbaum, Environ. Sci. Technol. 34 (2000) 1292.

[23] J.C. Rotticci-Mulder, M. Gustavsson, M. Holmquist, K. Hult, M. Martinelle, Protein Expr. Purif. 21 (2001) 386.

[24] P. Tomme, A. Boraston, B. McLean, J. Kormos, A.L. Creagh, K. Sturch, N.R. Gilkes, C.A. Haynes, R.A. Warren, D.G. Kilburn, J. Chromatogr. B, Biomed. Sci. Appl. 715 (1998) 283.

[25] M.A. Lemos, J.A. Teixeira, M. Mota, M. Gama, Biotechnol. Lett. 22 (2000) 703.

[26] M. Linder, I. Salovuori, L. Ruohonen, T.T. Teeri, J. Biol. Chem. 271 (1996) 21268.

[27] M. Linder, K. Selber, T. Nakari-Setälä, M. Qiao, M.-R. Kula, M. Penttilä, Biomacromolecules 2 (2001) 511.

[28] G.Y. Xu, E. Ong, N.R. Gilkes, D.G. Kilburn, D.R. Muhandiram, M. Harris-Brandts, J.P. Carver, L.E. Kay, T.S. Harvey, Biochemistry 34 (1995) 6993.

[29] D. Leckband, S. Sivanskar, Colloids Surf. B, Biointerfaces 14 (1999) 83. 